\title{
Side effects of incardronate disodium compared to pamidronate disodium in the treatment of bone metastasis pain: a systematic review and meta-analysis
}

\author{
Yang Wang ${ }^{1 \#}$, Zuolei Tang ${ }^{2 \#}$, Rongqin Meng ${ }^{1}$, Liang $\mathrm{He}^{1}$ \\ ${ }^{1}$ Department of Oncology, The Affiliated Chengdu 363 Hospital of Southwest Medical University, Chengdu, China; ${ }^{2}$ Department of Anesthesiology, \\ The Affiliated Chengdu 363 Hospital of Southwest Medical University, Chengdu, China \\ Contributions: (I) Conception and design: Y Wang; (II) Administrative support: L He; (III) Provision of study materials or patients: Z Tang; (IV) \\ Collection and assembly of data: R Meng; (V) Data analysis and interpretation: Y Wang; (VI) Manuscript writing: All authors; (VII) Final approval of \\ manuscript: All authors. \\ "These authors contributed equally to this work and should be considered as co-first authors. \\ Correspondence to: Liang He. Department of Oncology, The Affiliated Chengdu 363 Hospital of Southwest Medical University, Chengdu 610041, \\ China. Email: 16213218@QQ.com.
}

\begin{abstract}
Background: Bone metastasis is a common complication in patients with advanced malignant tumors and seriously impairs the quality of life of patients. Bisphosphonates are effective drugs for the treatment of bone metastasis pain. Incardronate disodium belongs to the 3rd generation of bisphosphonates. The efficacy and safety of bisphosphonates were explored using a systematic review and meta-analysis.

Methods: The databases PubMed (2000 to August 2021), EMBASE (2000 to August 2021), Cochrane library (August 2021), and CNKI (China National Knowledge Infrastructure, 2000 to August 2021) were searched. Randomized controlled studies involving patients being treated with incardronate for bone metastasis pain were included in the literature search. After screening and risk of bias assessment based on the Cochrane Handbook for Systematic Reviews of Interventions, Stata 16.0 software was used for analysis.

Results: The seven articles included in this study involved a total of 510 patients. Meta-analysis showed that there was no significant difference between incardronate and pamidronate disodium in the effectiveness of treating bone metastasis pain [odds ratio $(\mathrm{OR})=1.03,95 \%$ confidence interval (CI): 0.78-1.34, Z=0.188, $\mathrm{P}=0.851)$. The incidence of febrile adverse reactions from incardronate was significantly lower than pamidronate disodium $(\mathrm{OR}=0.58,95 \% \mathrm{CI}$ : 0.39-0.86, $\mathrm{Z}=-2.727, \mathrm{P}=0.006)$. The total incidence of adverse reactions from incardronate was significantly lower than pamidronate disodium (OR $=0.58,95 \%$ CI: 0.40 $0.85, \mathrm{Z}=-2.851, \mathrm{P}=0.004)$.

Discussion: The use of incardronate in the treatment of bone metastasis pain due to malignant tumors had comparable efficacy to the second-generation bisphosphonate pamidronate disodium. However, incardronate had fewer adverse reactions than pamidronate disodium.
\end{abstract}

Keywords! Incardronate; pamidronate disodium; bisphosphonates; bone metastases; pain

Submitted Sep 30, 2021. Accepted for publication Nov 09, 2021.

doi: 10.21037/apm-21-3056

View this article at: https://dx.doi.org/10.21037/apm-21-3056

\section{Introduction}

Bone metastasis is a common complication in patients with advanced malignancies, especially in lung cancer, breast cancer, prostate cancer, and nasopharyngeal carcinoma (1).
Some previous studies (2) have shown that the incidence of bone metastasis in late-stage breast cancer is as high as $65-75 \%$, while the incidence of bone metastasis in patients with lung cancer reaches $30-40 \%$. After the occurrence of 
bone metastasis, patients present with a series of osteolytic diseases, which can lead to bone pain, fracture, functional disorders, hypercalcemia, and other symptoms. The most typical symptom is stubborn pain, which seriously impacts the quality of life of patients (3). Clinically, it is necessary to actively control bone metastasis pain. In addition to surgery, chemoradiotherapy, immunotherapy, targeted drugs, and other treatments for the primary tumor, bisphosphates have been found to be effective for treating bone metastasis pain (4). Incardronate disodium belongs to the $3 \mathrm{rd}$ generation of bisphosphonates, which was one kind of incadronate acid, initially developed in 1997 and marketed in Japan. The antiresorptive strength of incardronate disodium is 1,000 times that of the $1 \mathrm{st}$ generation bisphosphonate, chlordronate, it has been shown in some studies to be long acting, rapid, and an efficient bone resorption inhibitor, with fewer toxic side effects compared with other bisphosphonates used in the treatment of osteoporosis, deformable osteoarthritis, hypercalcemia and bone pain caused by bone metastasis of malignant tumor (not including primary bone cancer itself) (5). A study (6) has reported the side effects between other kinds of third-generation (zoledronic acid) and second-generation bisphosphate (pamidronate disodium) in the treatment of bone metastasis pain, however, there is a lack of systematic evaluation of the effectiveness and side effects compare between incardronate and pamidronate disodium. This study analyzed the utility and safety of incardronate disodium to provide a basis for clinical practice.

We present the following article in accordance with the PRISMA reporting checklist (available at https://dx.doi. org/10.21037/apm-21-3056).

\section{Methods}

\section{Criteria for inclusion of literature in the study}

\section{Literature type}

The literature included in this study involved randomized controlled trials (RCTs), single-center and multi-center trials, and unlimited publication language. Controlled clinical trials (quasi-RCTs) and non-randomized concurrent controlled trials were excluded.

\section{Participants}

As humans were the research object of the literature search, studies involving rabbits, dogs, or rats were excluded. The selected patients had bone metastases from malignant tumors, confirmed by pathology and cytology, and the primary malignant tumor (breast cancer, intestinal cancer, gastric cancer, lung cancer, nasopharyngeal carcinoma, etc.) was irrelevant. Patients were not in a chemotherapy or radiotherapy cycle and were expected to have a life expectancy of $>6$ months. The imaging examination showed bone metastases and was accompanied by a moderate or higher pain score based on a numeric rating scale (NRS). There was no loss of heart, liver, kidney, or other major organ function during treatment.

\section{Description of intervention}

At least two groups of intervention methods for pain were required, including an experimental group using incardronate disodium and a control group using pamidronate disodium. Treatment and observation time were more than 3 weeks.

\section{Outcome indicators}

Primary outcome indicators included degree of pain reduction or effective rate of pain treatment, and adverse reaction rate.

Secondary outcome indicators were improvement in quality of life, serum calcium level, and serum phosphorus level.

\section{Search strategy and literature identification}

Search databases included PubMed (2000 to August 2021), EMBASE (2000 to August 2021), the Cochrane library (August 2021), and CNKI (China National Knowledge Infrastructure, 2000 to August 2021). The input keywords were: (incardronate/YM175) AND (pamidronate/ pamidronic) OR (Bisphosphonate) OR (bone metastases).

\section{Literature screening and data extraction}

Once the literature had been retrieved, Endnote X9 software was used for data management. After duplicates were excluded using the software's de-duplication function, two researchers independently completed the screening of included studies. Ineligible studies were identified and excluded by reading the title and abstract. After obtaining the original text and data, the remaining studies were further screened. If there was a conflict of opinion between the two researchers, a $3 \mathrm{rd}$ researcher was consulted to resolve the difference of opinion. 
Two researchers independently extracted data including:

(I) Basic information of literature: title, author, contact address, name of publication, and publication date;

(II) Basic characteristics of study: total number of samples, number of groups, and number of samples in each group;

(III) Basic characteristics of the participants: age of participants, gender, type of primary tumor, grade of bone pain, and presence of hypercalcemia;

(IV) Characteristics of intervention: different intervention methods used in the experimental group and the control group;

(V) Results evaluation: degree of pain relief, quality of life, and type and number of adverse reactions.

\section{Literature bias and evaluation analysis}

The Cochrane Handbook for Systematic Reviews of Interventions was used to assess the risk of bias for RCT studies, with high, low, or unclear indicating the risk of each dimension. We ranked an RCT "Level A" quality if all six aspects of the intervention were assessed with low risk of bias; If there was one or more "unclear risk of bias", it was ranked with Level B quality; If there was one or more "high risk of bias", it was ranked with Level C quality.

\section{Handling of data loss}

If a study did not include data but there was an access link provided, the data were obtained using the link. If there was no data at all, the original author was contacted to obtain the data, and if the data could not be obtained, the study was excluded.

\section{Statistical analysis}

\section{Effect measurement}

Odds ratio (OR) and 95\% confidence interval (CI) were used to assess binary variables (pain response rate and incidence of adverse reactions). $\mathrm{P}<0.05$ was considered to be statistically significant.

\section{Synthetic analysis tools and heterogeneity detection}

Stata 16.0 software was used for analysis, and forest plot was used to present the results of analysis. $\mathrm{I}^{2}$ and $\mathrm{Q}$ tests were used to analyze the heterogeneity of literature. $\mathrm{I}^{2}>50 \%$ or $\mathrm{P}<0.1$ indicated statistically significant heterogeneity.
Analysis of publication bias

Funnel plots were used to represent publication bias.

Heterogeneity survey and sensitivity analysis

The labbe function provided by Stata 16.0 was used to investigate heterogeneity, and the influence analysis tool was used for sensitivity analysis.

\section{Results}

\section{Literature search results and screening process}

The initial literature search identified 130 documents. After de-duplication and screening, a total of seven articles were included in the meta-analysis. Figure 1 shows the literature search results and screening process.

\section{Basic characteristics of the included literature}

The seven studies included in the meta-analysis involved a total of 510 patients (Table 1).

\section{Risk assessment of bias of included literature}

The risk of bias was assessed based on Cochrane (Table 2). One study (8) referred to grouping according to order (not random). The other studies did not mention grouping randomization or provide a specific random sequence generation method. None of the studies mentioned allocation concealment or blind methods. However, most set observation nodes and provided detailed descriptions for drop-out cases. No selective reporting or other bias was found.

\section{Comparison of the therapeutic efficacy of incardronate and pamidronate disodium}

All studies reported an effectiveness rate, including 253 patients treated with incardronate disodium and 255 patients treated with pamidronate disodium. As shown in Figure 2, meta-analysis revealed that there was no significant difference between incardronate and pamidronate disodium in effectiveness rate in the treatment of bone metastasis pain $(\mathrm{OR}=1.03,95 \%$ CI: $0.78-1.34, \mathrm{Z}=0.188, \mathrm{P}=0.851)$.

\section{Comparison of fever side effects of the 2 drugs}

All studies reported febrile adverse reactions during 
Identification of studies via databases and registers
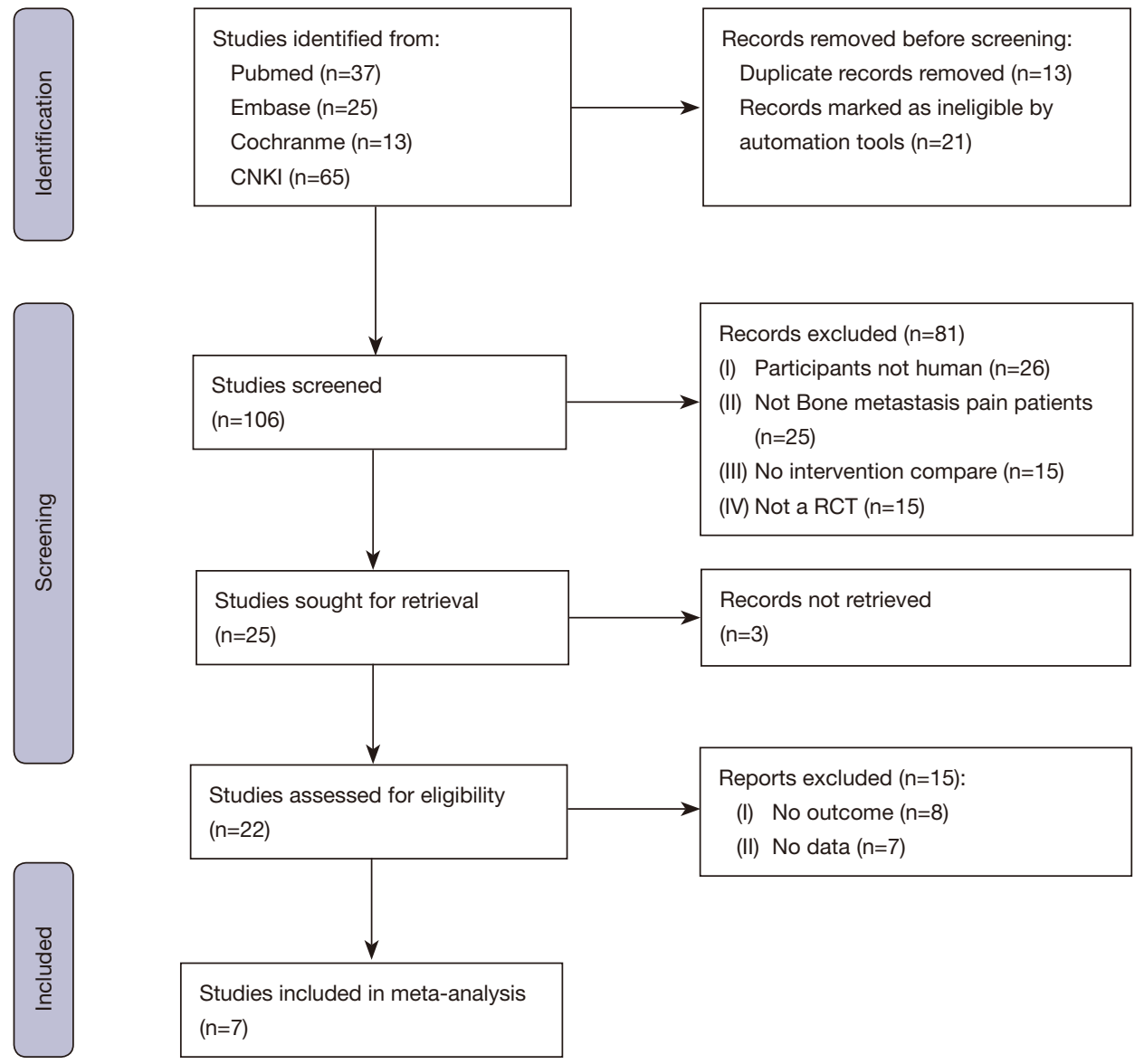

Figure 1 Literature screening flow chart.

treatment. The combined analysis results showed that the incidence rate of febrile adverse reactions from incardronate disodium was significantly lower than pamidronate disodium $(\mathrm{OR}=0.58,95 \%$ CI: $0.39-0.86, \mathrm{Z}=-2.727, \mathrm{P}=0.006)$ (Figure 3).

\section{Comparison of total adverse reactions of the two drugs}

All studies reported the number of adverse reactions during treatment. The combined analysis results showed that the total incidence rate of adverse reactions from incardronate disodium was significantly lower than pamidronate disodium $(\mathrm{OR}=0.58$, 95\% CI: $0.40-0.85, \mathrm{Z}=-2.851, \mathrm{P}=0.004$ ) (Figure 4).

\section{Heterogeneity investigation}

The Labbe plot showed there was no significant heterogeneity among the studies (Figure 5).

\section{Sensitivity analysis}

Sensitivity analysis showed that the seven studies had similar distribution on both sides and good stability, as shown in Figure 6.

\section{Analysis of publication bias}

The funnel plot showed that the left and right distributions of the 7 articles were basically symmetrical, without significant publication bias (Figure 7).

\section{Discussion}

Bone metastasis refers to the metastasis of the primary tumor into bone tissue through the bloodstream and lymph system, leading to dissolved or damaged bone tissue. 


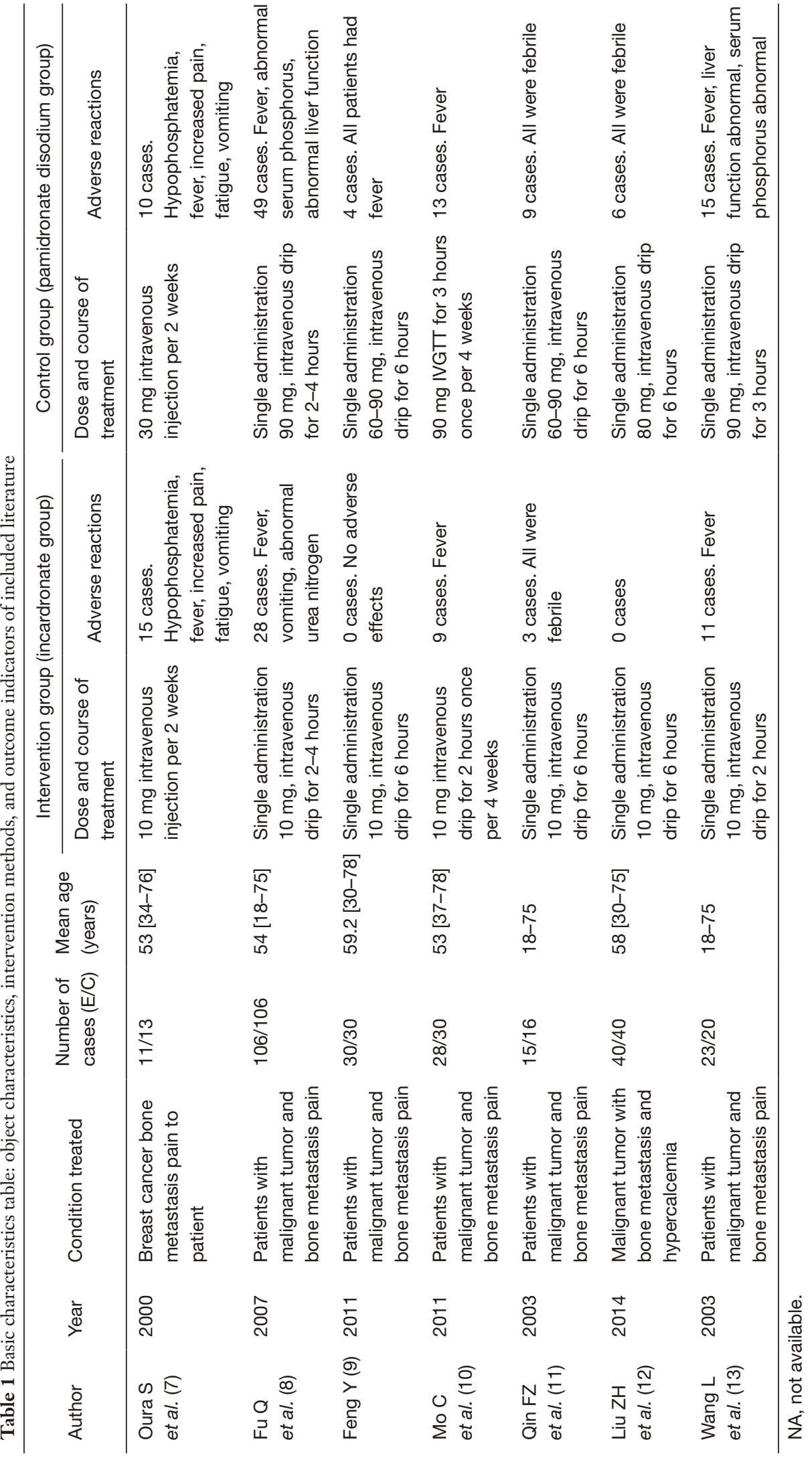


Table 2 Risk of bias and quality assessment based on the Cochrane Handbook for Evaluation of Randomized Interventions

\begin{tabular}{|c|c|c|c|c|c|c|c|}
\hline Study & $\begin{array}{c}\text { Random sequence } \\
\text { generation }\end{array}$ & $\begin{array}{l}\text { Classification } \\
\text { hiding }\end{array}$ & Blind method & Data integrity & $\begin{array}{l}\text { Optional } \\
\text { reporting }\end{array}$ & Other bias & Quality \\
\hline Oura S et al. (7) & Unclear & Unclear & Unclear & Low & Low & Low & Level B \\
\hline Feng Y (9) & Unclear & Unclear & Unclear & Low & Low & Low & Level B \\
\hline Liu ZH et al. (12) & Unclear & Unclear & Unclear & Unclear & Low & Low & Level B \\
\hline Wang L et al. (13) & Unclear & Unclear & Unclear & Low & Low & Low & Level B \\
\hline
\end{tabular}

\begin{tabular}{|c|c|c|c|}
\hline Study (year) & & $\begin{array}{l}\text { Odds ratio } \\
(95 \% \mathrm{Cl})\end{array}$ & $\begin{array}{c}\% \\
\text { Weight }\end{array}$ \\
\hline Oura S et al. (2000) & & $0.82(0.25,2.63)$ & 5.96 \\
\hline Fu Q et al. (2007) & & $1.03(0.67,1.57)$ & 40.50 \\
\hline Yu F et al. (2011) & & $1.04(0.49,2.19)$ & 12.94 \\
\hline Mo C et al. (2011) & & $1.41(0.61,3.22)$ & 9.03 \\
\hline Qin F Z et al. (2003) & & $0.89(0.30,2.66)$ & 6.51 \\
\hline Liu ZH et al. (2014) & & $0.97(0.51,1.86)$ & 17.72 \\
\hline Wang L et al. (2003) & & $0.95(0.34,2.62)$ & 7.34 \\
\hline Overall, $\mathrm{MH}\left(I^{2}=0.0 \%, \mathrm{P}=0.992\right)$ & & $1.03(0.78,1.34)$ & 100.00 \\
\hline $\begin{array}{c}1 \\
0.25\end{array}$ & 1 & & \\
\hline
\end{tabular}

Figure 2 Comparison of the effectiveness rate of treatment with incardronate and pamidronate disodium for bone metastasis pain.

Cancer cells can directly destroy the mineralized matrix of the bone structure by stimulating osteolysis $(14,15)$. Inhibition of bone destruction and reduction of bone pain are the basis for improving the quality of life of patients with advanced bone metastases. At present, there are many treatment methods for bone metastasis pain. Analgesics, chemoradiotherapy, hormone therapy, and other measures can reduce bone destruction and reduce pain. However, chemoradiotherapy alone can only produce local efficacy and cannot be used for the treatment of patients with systemic bone metastasis, while chemoradiotherapy can cause considerable adverse reactions (16). In recent years, the application of double silicates has brought new treatment methods to patients with multiple bone metastasis pain. The therapeutic principle is that they are directly absorbed to act on bone tissue, inhibit the activity of osteoclasts, slow down the destruction of bone structure, treat osteolysis associated with osteoclasts, and influence tumor-induced melting, osteoblastic, and mixed bone destruction $(17,18)$. In the study by Saad et al. (19), zoledronic acid was used in the treatment of advanced bone metastasis in patients with prostate cancer, resulting in good long-term results. Pamidronate disodium is a secondgeneration bisphosphonate drug, and its efficacy has been affirmed in numerous clinical studies (20). In this study, 253 patients were treated with incardronate disodium and 255 patients were treated with pamidronate disodium. Metaanalysis showed that there was no statistical difference in the effectiveness of the two drugs, implying that incardronate had similar efficacy to pamidronate disodium. Incardronate, a third-generation bisphosphate, can bind directly to bone matrix physicochemical properties, interfere with osteolysis 


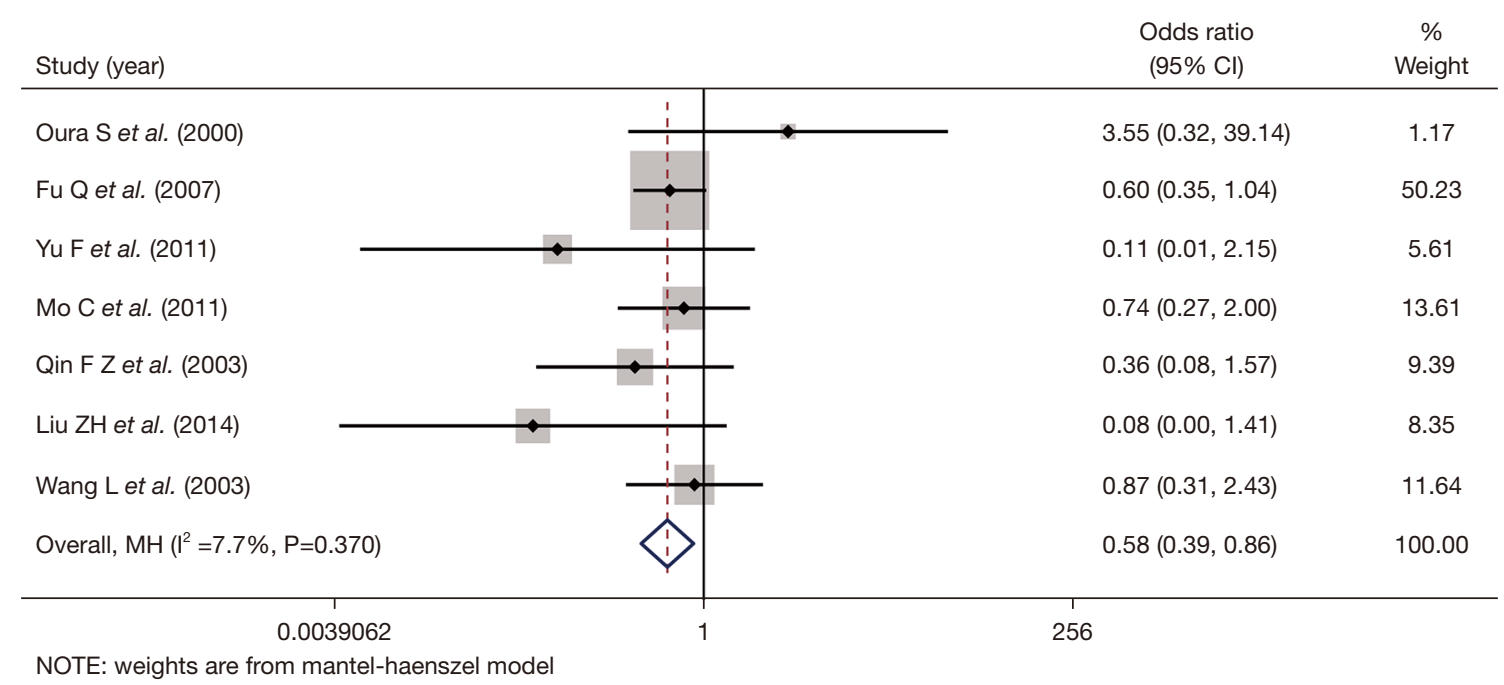

Figure 3 Comparison of fever side effects of incardronate and pamidronate disodium treatment.

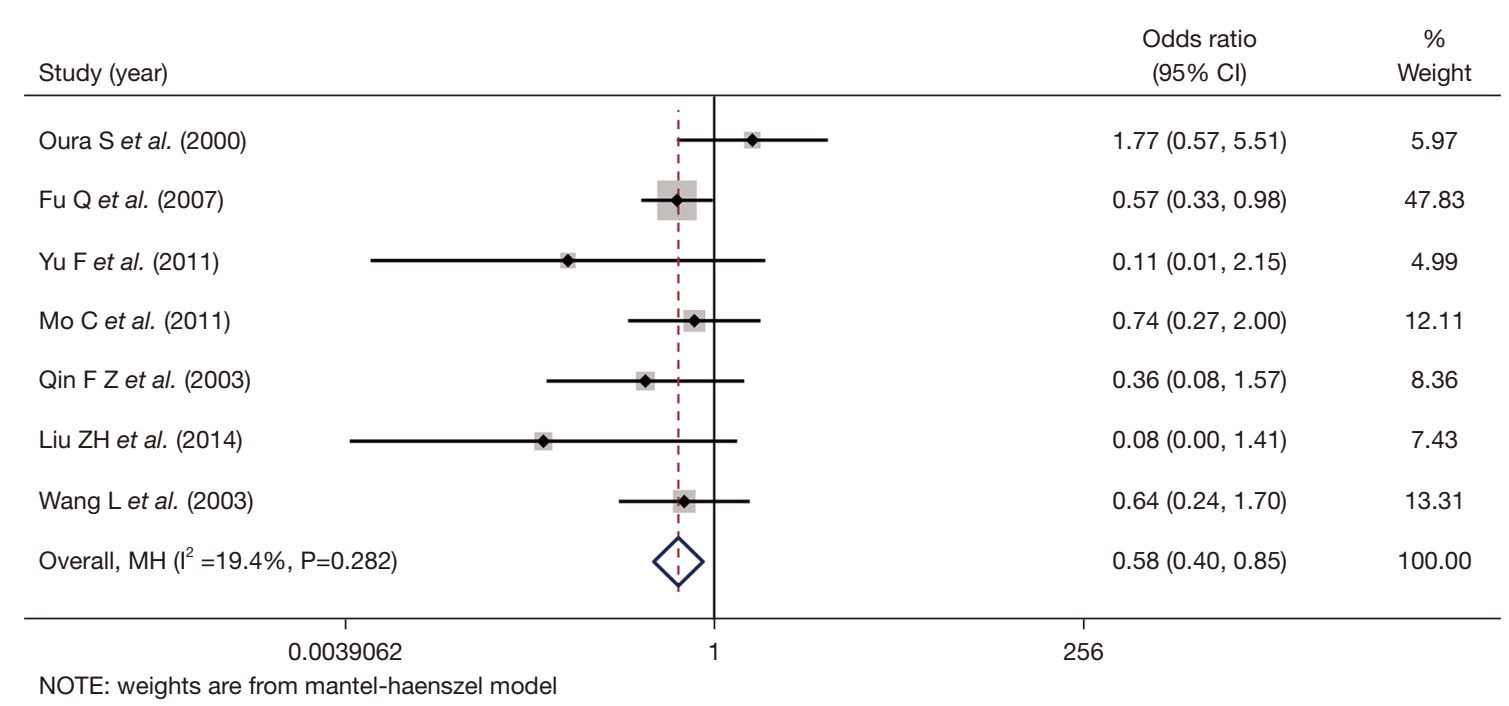

Figure 4 Comparison of adverse reactions of treatment with incardronate and pamidronate disodium.

and absorption, and prevent the adhesion of osteoclasts in bone tissue. Additionally, incardronate disodium can reduce the differentiation and proliferation of osteoclasts, induce their apoptosis, inhibit their number and activity, and impede the invasion of tumor cells, thereby reducing their colonization in bone tissue and controlling the spread of bone metastases (21).

All of the studies in this review reported the incidence of adverse reactions from the two drugs, with the combined effect size showing that the fever rate and total adverse reaction rate of incardronate disodium was less than pamidronate disodium. The main adverse reaction of treatment with incardronate disodium was fever $(8,10)$, which was relieved after antipyretic drug intervention. A small number of patients experienced nausea, vomiting, fatigue, and abnormal urea nitrogen, but there was no abnormal liver function or nephrotoxicity reported, indicating the safety of incardronate. The study (11) pointed out the incidence of other adverse reactions, such as fatigue and skeletal muscle pain is much lower, most of which occur 2-3 days after medication and can be relieved automatically or after some symptomatic treatment, and the molecular 
structure of incardronate makes it easier to be absorbed and can prevent the release of inflammatory mediators.

At present, the most commonly used bisphosphonates in clinical practice include pamidronate disodium, ibandronate sodium, zoledronic acid and incardronate disodium. Among them, ibandronate sodium, zoledronic acid and incardronate sodium belong to the third generation bisphosphonates. Studies $(22,23)$ have compared the therapeutic effects of incadronate disodium with other third generation

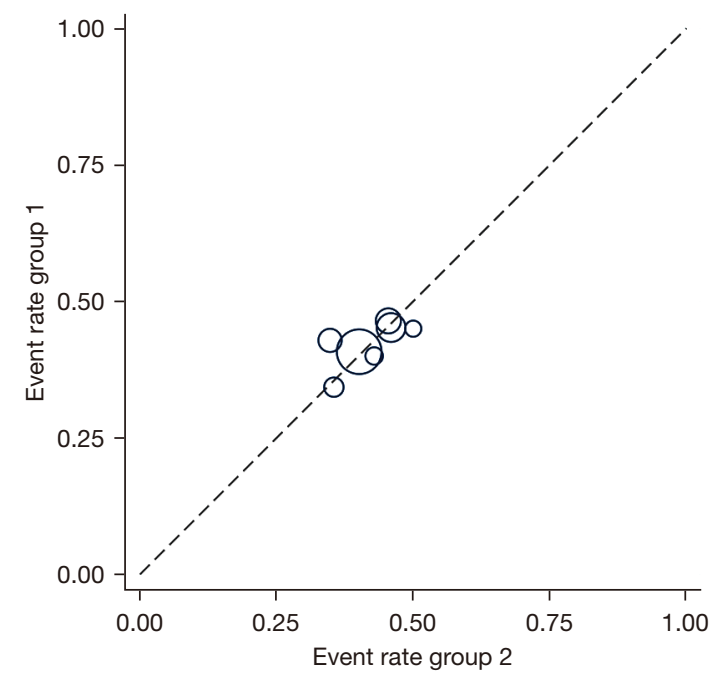

Figure 5 Labbe plot for heterogeneity. bisphosphonates, the results of which showed that the 14 days efficacy of incadronate disodium was up to $90.0 \%$, and most of the patients had good tolerance, no obvious adverse reactions, the safety and efficacy were better than other drugs. However, one study (24) compared the costs of ibandronate, zoledronic acid and incardronate disodium and found that the costs of the three drugs are sorted with zoledronic acid < ibandronate sodium < incadronate disodium at conventional doses. Therefore, zoledronic acid is still the main drug in clinical application.

In this study, the Labbe plot for heterogeneity showed that the literature was evenly distributed, as did the sensitivity analysis diagram, indicating that there was no significant heterogeneity between the studies. However, most of the studies did not mention the random sequence generation method, allocation concealment method or blind method, and thus the quality of the literature may bias the application of the results. In addition, the sample sizes of the included studies were small. Further controlled clinical studies with larger sample sizes and multiple centers are needed to provide stronger evidence for the efficacy and safety of incardronate compared to pamidronate disodium.

\section{Conclusions}

In summary, the application of incardronate for treating bone metastasis pain produced efficacy equivalent to the

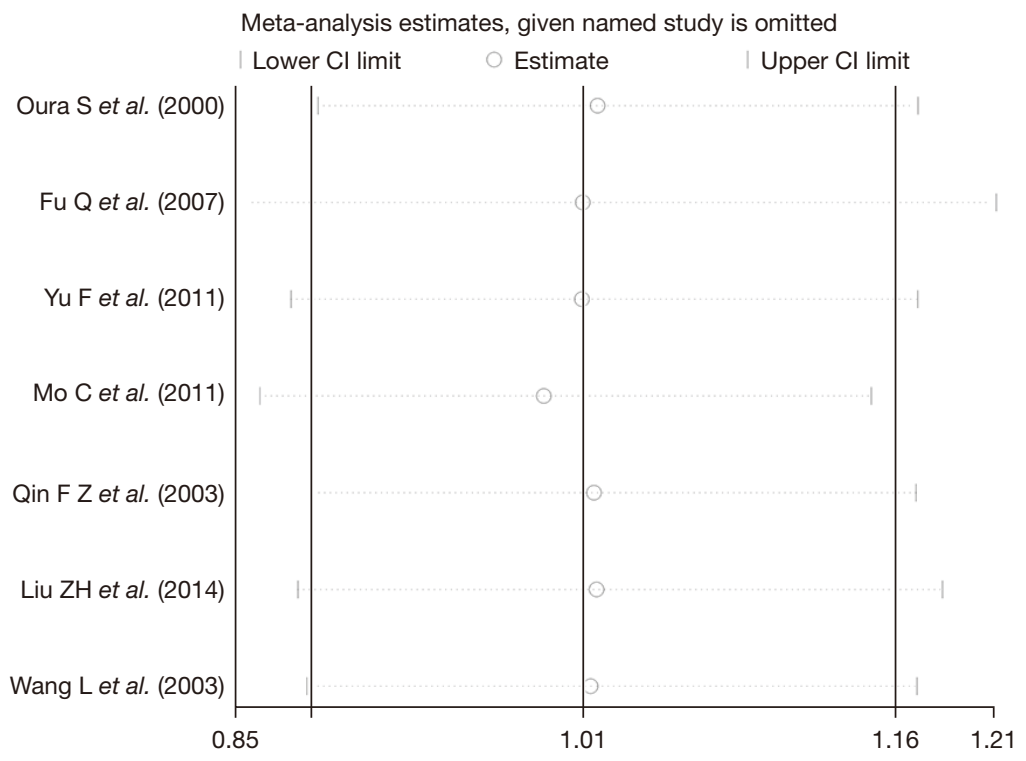

Figure 6 Sensitivity analysis. 


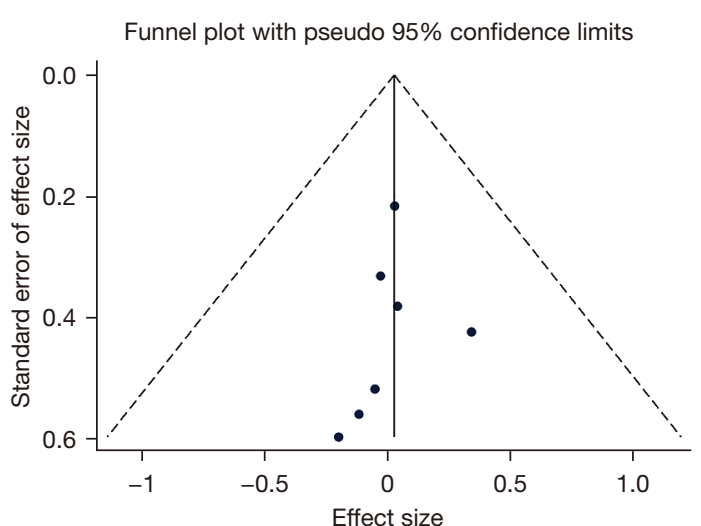

Figure 7 Funnel plot analysis.

second-generation bisphosphonate pamidronate disodium, but incardronate had far fewer adverse reactions than pamidronate disodium. As the sample size of this study was small, more RCTs of better quality should be included in future reviews to continue exploring the safety of incardronate.

\section{Acknowledgments}

Funding: None.

\section{Footnote}

Reporting Checklist: The authors have completed the PRISMA reporting checklist. Available at https://dx.doi. org/10.21037/apm-21-3056

Conflicts of Interest: All authors have completed the ICMJE uniform disclosure form (available at https://dx.doi. org/10.21037/apm-21-3056). The authors have no conflicts of interest to declare.

Ethical Statement: The authors are accountable for all aspects of the work in ensuring that questions related to the accuracy or integrity of any part of the work are appropriately investigated and resolved.

Open Access Statement: This is an Open Access article distributed in accordance with the Creative Commons Attribution-NonCommercial-NoDerivs 4.0 International License (CC BY-NC-ND 4.0), which permits the noncommercial replication and distribution of the article with the strict proviso that no changes or edits are made and the original work is properly cited (including links to both the formal publication through the relevant DOI and the license). See: https://creativecommons.org/licenses/by-nc-nd/4.0/.

\section{References}

1. Wood SL, Westbrook JA, Brown JE. Omic-profiling in breast cancer metastasis to bone: implications for mechanisms, biomarkers and treatment. Cancer Treat Rev 2014;40:139-52.

2. Ye LJ, Suo HD, Liang CY, et al. Nomogram for predicting the risk of bone metastasis in breast cancer: a SEER population-based study. Transl Cancer Res 2020;9:6710-9.

3. Yang M, Yu X. Management of bone metastasis with intravenous bisphosphonates in breast cancer: a systematic review and meta-analysis of dosing frequency. Support Care Cancer 2020;28:2533-40.

4. Milner RJ, Farese J, Henry CJ, et al. Bisphosphonates and cancer. J Vet Intern Med 2004;18:597-604.

5. Li C, Mori S, Li J, et al. Long-term effect of incadronate disodium (YM-175) on fracture healing of femoral shaft in growing rats. J Bone Miner Res 2001;16:429-36.

6. Yang L, Du S. Efficacy and Safety of Zoledronic Acid and Pamidronate Disodium in the Treatment of Malignant Skeletal Metastasis: A Meta-Analysis. Medicine (Baltimore) 2015;94:e1822.

7. Oura S, Tanino H, Yoshimasu T, et al. Bisphosphonate therapy for bone metastases from breast cancer: clinical results and a new therapeutic approach. Breast Cancer 2000;7:307-10.

8. Fu Q, Wang YJ, Qin SK, et al. Phase II clinical trial of home-made incardronate in patients with pain of bone metastasis of malignancy or/and hypercalcemia. Pharmaceutical Care and Research 2007;3:180-3.

9. Feng Y. Clinical study of incardronate in the treatment of bone metastases. China Drug Application and Monitoring 2011;8:200-2.

10. Mo C, Huang YP, Wu XE, et al. Efficacy and Safety of Incadornate for Malignant Osseous Metastasis Pain. Evaluation and Analysis of Drug-Use in Hospitals of China 2011;11:549-51.

11. Qin FZ, Zheng RS, Zhai, YZ, et al. incardronate in the treatment of pain in 15 patients with metastatic tumor of bone. Chinese New Drugs Journal 2003;(5):380-2.

12. Liu ZH, Yang CB, Yu RB. The clinical observation of two sodium for treating breast cancer bone metastases. China Prescription Drug 2014;12:65.

13. Wang L, Qin SK, Chen YX, et al. Phase II clinical trial of 
domestic dicadronate in the treatment of pain from bone metastases. J Clin Oncol 2003;(03):204-7.

14. Aielli F, Ponzetti M, Rucci N. Bone Metastasis Pain, from the Bench to the Bedside. Int J Mol Sci 2019;20:280.

15. Miaskowski C, Lee KA. Pain, fatigue, and sleep disturbances in oncology outpatients receiving radiation therapy for bone metastasis: a pilot study. J Pain Symptom Manage 1999;17:320-32.

16. Steenland E, Leer JW, van Houwelingen H, et al. The effect of a single fraction compared to multiple fractions on painful bone metastases: a global analysis of the Dutch Bone Metastasis Study. Radiother Oncol 1999;52:101-9.

17. Heidenreich A, Hofmann R, Engelmann UH. The use of bisphosphonate for the palliative treatment of painful bone metastasis due to hormone refractory prostate cancer. J Urol 2001;165:136-40.

18. Costa L, Major PP. Effect of bisphosphonates on pain and quality of life in patients with bone metastases. Nat Clin Pract Oncol 2009;6:163-74.

19. Saad F, Gleason DM, Murray R, et al. Long-term efficacy of zoledronic acid for the prevention of skeletal complications in patients with metastatic hormone-refractory prostate cancer. J Natl Cancer Inst

Cite this article as: Wang Y, Tang Z, Meng R, He L. Side effects of incardronate disodium compared to pamidronate disodium in the treatment of bone metastasis pain: a systematic review and meta-analysis. Ann Palliat Med 2021;10(11):1195011959. doi: 10.21037/apm-21-3056
2004;96:879-82.

20. Koizumi M, Kobayashi M, Furukawa M, et al. The bisphosphonate incardronate for bone metastases of breast cancer. Int J Clin Oncol 2000;5:241-6.

21. Nishikawa M, Yamamoto M, Murakami T, et al. A thirdgeneration bisphosphonate, YM175, inhibits osteoclast formation in murine cocultures by inhibiting proliferation of precursor cells via supporting cell-dependent mechanisms. J Bone Miner Res 1998;13:986-95.

22. Lan PL, Meng LX. Clinical study of incadronate disodium in the treatment of osteoporosis. Practical Drugs and Clinic 2011;14:123-5.

23. Wang JF, Li ZL. Review on the efficacy and safety of nitrogenous bisphosphonates in the treatment of bone metastasis of malignant tumors. China Prescription Drug 2020;18:20-1.

24. Song JF, Zhu H, Han S. Cost effectiveness analysis of ibandronate sodium, zoledronic acid and incadronate disodium in the treatment of bone metastasis of malignant tumors. Chinese Pharmacoeconomics 2019;14:9-15.

(English Language Editor: A. Muijlwijk) 